\title{
Fluorescence-lifetime imaging with a multifocal two-photon microscope
}

\author{
S. Lévêque-Fort and M. P. Fontaine-Aupart \\ Laboratoire de Photophysique Moléculaire, Centre National de la Recherche Scientifique, Unité Propre de Recherche 3361, Centre \\ Universitaire, Batiment 210, 91405 Orsay, France

\section{G. Roger and P. Georges} \\ Laboratoire Charles Fabry de l'Institut d'Optique, Centre National de la Recherche Scientifique and Université Paris Sud, Unité \\ Mixte de Recherche 8501, Centre Universitaire, Batiment 503, 91405 Orsay, France
}

Received June 30, 2004

\begin{abstract}
Two-photon microscopy is a powerful tool for imaging of cells or tissues. However, it presents the drawback of being a laser-scanning technique that involves a long acquisition time for fluorescence-lifetime imaging. Thus it is commonly limited to intensity images that give only indications of the location of fluorophores but do not identify the physicochemical properties and interactions between cells' components. To protect biological samples from experiments that are too long and to provide a more comprehensive spectroscopic tool we have developed a time-resolved multifocal multiphoton microscope. This setup allows us to speed up the acquisition while retaining the possibility of measuring both intensity and lifetime images of the sample. (C) 2004 Optical Society of America
\end{abstract}

OCIS codes: $180.2520,170.3650,190.4180$.

The fluorescence lifetime is a powerful spectroscopic tool that is successfully applied in several domains such as medicine, biology, and chemistry. Its high sensitivity can be used to probe locally the influence of the environment, to distinguish among chemical species, and to detail fast photochemical processes. Combined with two-photon microscopy, lifetime measurement directly benefits from all the advantages associated with multiphoton excitation compared with conventional confocal laser-scanning microscopy: inherent three-dimensional imaging can be performed without the need for a confocal pinhole, photobleaching and photodamage are greatly reduced and are confined to the focal volume, and the near-infrared lasers typically employed penetrate more deeply than visible-light lasers into tissue, as scattering and absorption are reduced.

One of the main drawbacks of a conventional two-photon microscope is its imaging collection rate. As it is a laser-scanning technique in which images are acquired point by point, it rapidly becomes time consuming to acquire a fluorescence-lifetime image (FLIM) of the whole sample. Of course the acquisition time depends strongly on both the fluorescent quantum yield of fluorophores and the signal-to-noise ratio needed for analyzing the decay data. For example, to acquire a FLIM image with a time-correlated single-photon counting system, an acquisition time of $1 \mathrm{~s} /$ point permits a good signal-to-noise ratio to be attained, but it takes $\sim 200$ min to obtain a FLIM map $(100 \mu \mathrm{m} \times 120 \mu \mathrm{m}$; step width, $1 \mu \mathrm{m})$. Acquisition of a fluorophore's lifetime is then usually made for a few interesting sample points.

Several ways to speed up acquisition time, such as increasing the fluorophore concentration or the excitation beam's intensity, have been tried but most of them are not appropriate for biological studies. The best way to increase the imaging speed of a biological system is to illuminate several points of the sample simultaneously. The acquisition time is thus reduced by at least the number of excitation beams used. The principle of multifocal multiphoton microscopy (MMM) has been demonstrated by several groups of scientists. Various approaches to generating multifocal points have been developed: e.g., use of a microlens disk, ${ }^{1}$ of a cascade of beam splitters, ${ }^{2}$ or of a beam splitter associated with multiple mirrors. ${ }^{3,4}$ MMM requires important changes for detection compared with the single-beam approach: a photomultiplier can no longer be used, as a detector that is capable of detecting the fluorescence emitted from each focus is needed. Typically, a CCD camera with high sensitivity is used. Time-resolved measurements can be obtained for all the foci by integration of an ultrafast optical gated intensifier in front of the CCD camera, but it has been investigated only for the microlens approach. ${ }^{5}$ We report here our time-resolved MMM system based on a homemade beam generator associated with a CCD camera and an optical gated intensifier that permits dynamic measurement of fluorescence with a typical resolution of $25 \mathrm{ps}$, to our knowledge the first time that it has been demonstrated with this approach.

Our multifocal beam generator, depicted in Fig. 1, is composed of four high-reflectivity mirrors and a $50 \%$ beam splitter (Suprasil, $60 \mathrm{~mm} \times 40 \mathrm{~mm} \times 2 \mathrm{~mm}$, $p$ polarization). The initial beam is separated into two beams by the $50 \%$ beam splitter. The ensuing two beams are reflected back, respectively, by mirrors M1 and M2 of the beam generator; the result is four beams. The reflection of the four beams on the beam splitter doubles the number of beams. We then obtain eight beams in the same plane, separated by distances imposed by the locations of mirrors M1-M4 with respect to the beam splitter. In these conditions 


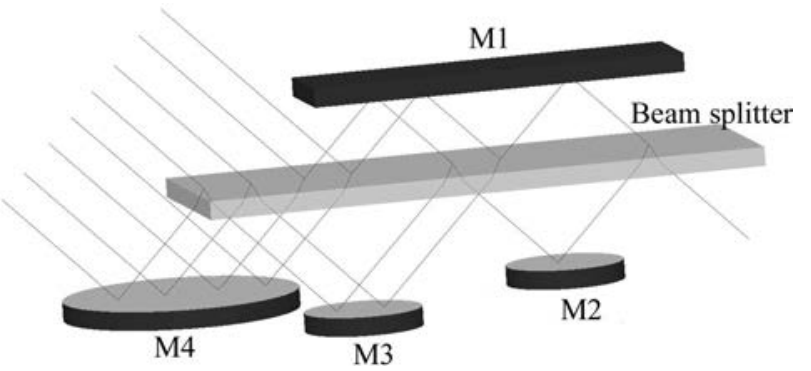

Fig. 1. Schematic of the multifocal beam generator.

we can easily change the distance between beams $(3-6 \mu \mathrm{m})$, which is not allowed in a commercial system. The last point is interesting specifically when one is investigating diffusing samples or simply when one is working several tens of micrometers inside biological samples; fluorescence is scattered and, by adjusting the distance between foci, one can avoid cross talk at the detection site. Cross talk at the excitation site can also be a major issue when the foci are close together; the out-of-focus tails of neighboring foci start to generate a significant amount of two-photon-induced fluorescence. ${ }^{6,7}$ The use of a beam generator prevents this problem; differences in optical path lengths induces an intrinsic delay of a few picoseconds between the beams, and thus no cross talk exists even if the beams are close spatially. The overall transmission of the beam generator was $90 \%$, which is much higher than for the microlens disk approach to generating multiple beams. By measuring each beam power we observed a maximum deviation of $18 \%$ from the mean value. This nonuniformity is due mainly to small differences between the mirrors' reflectivities and to the transmission of the 50-50\% beam splitter, which is not exactly 0.5 but fluctuates slowly with the laser excitation wavelength. Combined with high-numerical-aperture objectives, all beams can generate diffraction-limited foci and thus exhibit spatial resolution similar to that of a classic two-photon microscope. To preserve the sample we reduced the power of each focus to $1-10 \mathrm{~mW}$, as is typically used for single-beam excitation.

Our two-photon system is based on a homemade inverted microscope, as depicted in Fig. 2. By using two flip-flap mirrors we made it possible to study samples in either of two complementary modes: with classical wide-field one-photon excitation (OPE) or with multifocal two-photon excitation (MTPE). The laser beam delivered by a Ti:sapphire (Ti:Sa) laser (Mira from Coherent; $800 \mathrm{~mW}, 76 \mathrm{MHz}, 100 \mathrm{fs}, 800 \mathrm{~nm}$ ) is either frequency doubled with a $\beta$-barium borate (BBO) or divided into a line of eight beams by the beam generator described above. To create eight separated foci in the focal plane requires the beams to enter the objective at slightly different angles. We then tilt mirrors M2-M4 to make the eight beams converge at one point. This point is located in the focal plane of the first lens of a telescope ( $f 1=100 \mathrm{~mm} ; f 2=400 \mathrm{~mm}$ ), which also corresponds to the position of the $X Y$ scanning mirrors used to move the eight beams on the sample simultaneously to reconstruct a whole image. The telescope is adjusted first to increase the entire beam diameter to cover the back aperture of the objective (immersion oil, Zeiss Planeofluar $63 \times$; N.A., 1.4) and produce eight diffraction-limited excitation spots and second to increase all the values of the angles between the beams. The fluorescence emitted from each excited point is collected by the same objective. Each fluorescent point is imaged, via the dichroic mirror and a filter to block the excitation light, onto a high rate imager (HRI; Kentech Instruments, Ltd.), which is capable of sampling the fluorescence at a repetition rate of $76 \mathrm{MHz}$, with a minimum gate time of $200 \mathrm{ps}$, in 25-ps steps. The imager is read out by a 12-bit CCD camera (Hamamatsu Model Orca ER) that is optically coupled to the intensifier using two standard camera lens objectives ( $f=50 \mathrm{~mm})$. The CCD camera is used in binning mode $(2 \times 2$ pixels with one $6.45 \mu \mathrm{m} \times 6.45 \mu \mathrm{m}$ pixel), which allows us to speed up the readout time (16.4 frames/s) without compromising the spatial resolution. One can measure the fluorescence lifetime in the time domain by sampling the fluorescence emitted following an excitation pulse. Whole-field FLIM measurement with one-photon excitation has been successfully made by means of a high-rate imager coupled to a CCD camera; it exhibits resolution down to $10 \mathrm{ps}$ at $10 \mathrm{kHz} .{ }^{8}$ This detection approach has been successfully applied to our MMM setup, in which spatial and temporal resolution of the emitted fluorescence is needed for the eight foci. We then acquire a whole series of time-gated intensity images by triggering the imager at different delays after the excitation pulse. Typically, a set of images at 7-20 delay times is acquired. Using the Levenberg-Marquardt nonlinear least-squares fitting algorithm, we then fit each pixel in the image set to a monoexponential decay. The calculated lifetimes are displayed as a lifetime map with a false color scale. The acquisition time needed to obtain a MTPE FLIM is decreased not only by the number of excitation points but also by this specific detection scheme. Sampling the fluorescence at different delays rather than applying a photon-counting approach strongly reduces the acquisition time, which thus depends mainly on the number of time-gated intensity images needed for proper analysis of the fluorescence decay. To reconstruct the fluorescence intensity and the FLIM of a cell or a tissue we then

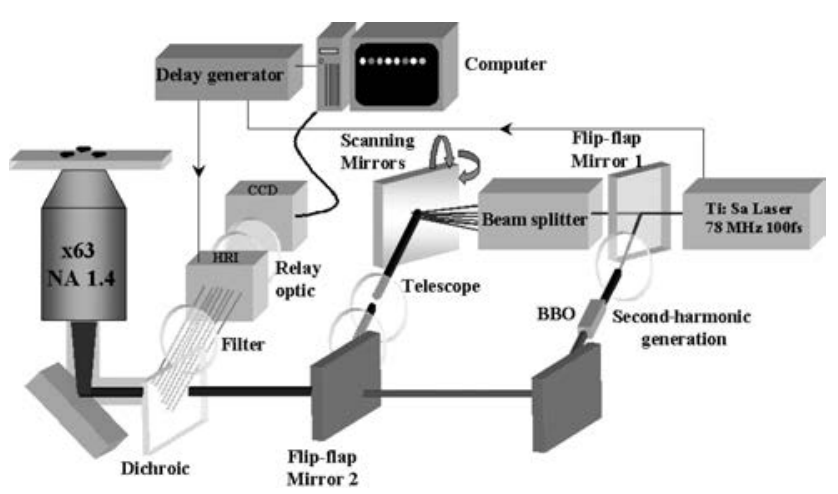

Fig. 2. Experimental setup of the time-resolved multiphoton microscope associated with a wide-field one-photon microscope. 


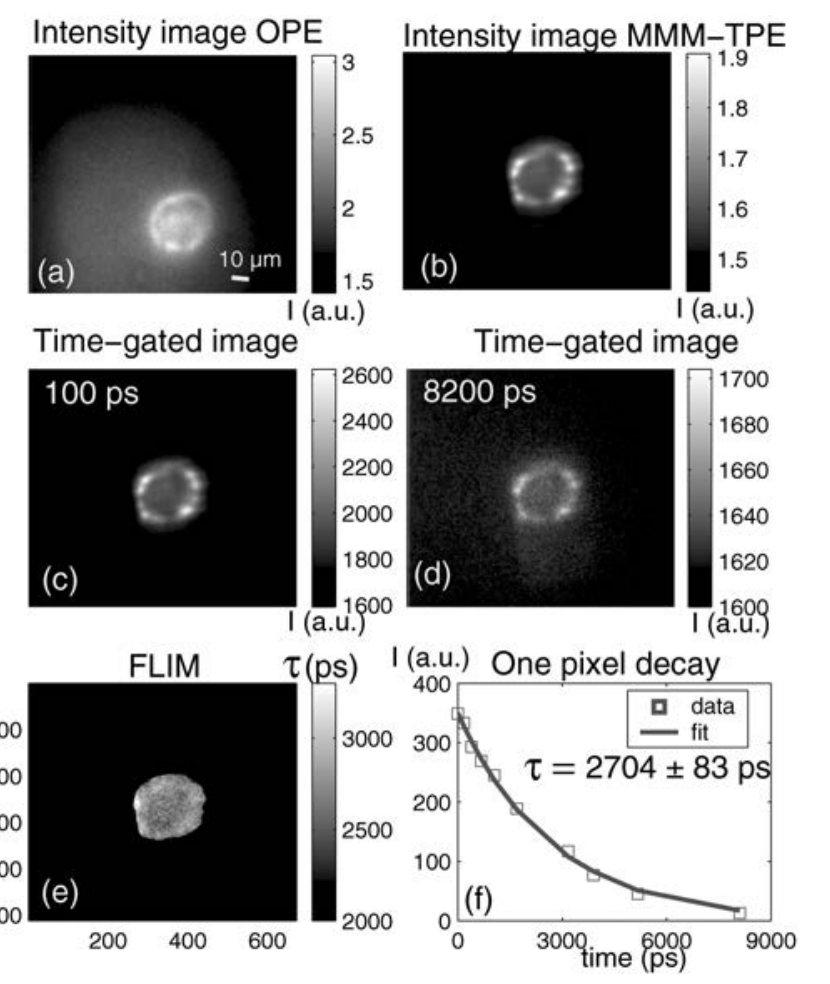

Fig. 3. Autofluorescence images obtained on a urothelial cell: (a) intensity image under $\mathrm{OPE}$, (b) intensity image under MTPE. Time-gated intensity images (c) $100 \mathrm{ps}$ and (d) $8200 \mathrm{ps}$ after the laser pulse. (e) FLIM under MTPE and (f) one typical fluorescence decay.

need uniform and simultaneous scanning of the eight foci in the focal plane of the sample. The displacement of the foci with the scanning mirrors is carefully calibrated by investigation of a homogeneous sample, typically a monolayer of Rhodamine $\mathrm{B}$, which allows us to avoid overexposed areas caused by nonoptimal scanning steps. Depending on the fluorophore's quantum yield, each point of the sample is illuminated $0.5-10 \mathrm{~ms}$; the intensity image is thus reconstructed by integration of the signal on the CCD during scanning of the whole sample. Even if this approach is not the best one in terms of signal-to-noise ratio, it directly gives the intensity image without any postprocessing treatment. One of the main goals of the MMM system is to improve cell measurements through both intensity and lifetime imaging. In Fig. 3 we present the results of our study of an autofluorescent urothelial cell studied under OPE and MTPE. The interfocal distance was chosen to be $6 \mu \mathrm{m}$ to prevent any cross talk at the detector, and for this sample a scanning step of $1 \mu \mathrm{m}$ was applied. Figures 3(a) and 3(b) correspond, respectively, to the fluorescence intensity images obtained under wide-field OPE and MTPE. Acquisition time parameters for MTPE are a 1-ms excitation time for each focal position, leading to a one intensity image in $1.5 \mathrm{~s}$. To compute the whole lifetime map of the cell we recorded ten intensity images, leading to an acquisition time of only $15 \mathrm{~s}$ (field of view, $100 \mu \mathrm{m} \times 120 \mu \mathrm{m}$ ). Under OPE the acquisition time was comparable (intensity image,
$1.8 \mathrm{~s} ;$ FLIM, $18 \mathrm{~s}$ ), at the cost of lower axial resolution, as expected for wide-field imaging. Similar samples were investigated with a single-point two-photon microscope, and a signal-to-noise ratio comparable to that obtained for MTPE but for total acquisition times of $12 \mathrm{~s}$ for the intensity image and $200 \mathrm{~min}$ for the FLIM was obtained. MTPE thus strongly reduced the acquisition time, by factors of 8 for the intensity image and of 800 for the FLIM. Time-gated intensity images are represented for MTPE at two delays of 100 ps [Fig. 3(c)] and 8200 ps [Fig. 3(d)] after the excitation pulse. Under MTPE the lifetime map is homogeneous [Fig. 3(e)]. The mean lifetime calculated over the whole cell is $2.7 \mathrm{~ns}$ (standard deviation, $0.15 \mathrm{~ns}$ ), which is less than for OPE (3.2 ns; data not presented here) and is certainly due to the efficient removal of the perturbing background surrounding the cell. We also point out that the small difference in intensity in the cytoplasm of the cell is due to small differences in the fluorophore's concentration, as the mean lifetime value calculated for each bright area is within the lifetime distribution calculated over the cell, except for one lifetime value, which is much closer to 3 ns. Figure 3(f) represents a typical fluorescence decay for one pixel under MTPE; we can see fairly good agreement between the data set and the monoexponential fit.

In summary, we have demonstrated the advantages of our homemade time-resolved multifocal multiphoton microscope, which delivers in a considerably reduced acquisition time both intensity and lifetime images without compromising axial resolution or inducing cross talk. A MTPE lifetime image on cells clearly demonstrated the relevance of this approach for a large number of biological applications.

This research has been partially funded by the Centre National de la Recherche Scientifique (Actions Concertees Incitatives Nanostructures-Nanosciences) and by the research program Pole Laser of the Contrat Plan Etat Region (2000-2006; French State and Conseil General de l'Essonne). S. Lévêque-Fort's e-mail address is sandrine.fort@ppm.u-psud.fr.

\section{References}

1. J. Bewersdorf, R. Pick, and S. H. Hell, Opt. Lett. 23, 655 (1998).

2. D. N. Fittinghoff, P. W. Wiseman, and J. Squier, Opt. Express 7(8), 273 (2000), http://www.opticsexpress.org.

3. T. Nielsen, M. Fricke, D. Hellweg, and P. Andresen, J. Microsc. 201, 368 (2001).

4. S. Lévêque-Fort, M. P. Fontaine-Aupart, G. Roger, and P. Georges, Proc. SPIE 5139, 173 (2003).

5. M. Straub and S. H. Hell, Appl. Phys. Lett. 73, 1769 (1998).

6. A. H. Buist, M. Muller, J. Squier, and G. J. Brakenhoff, J. Microsc. 192, 2176 (1998).

7. A. Egner and S. H. Hell, J. Opt. Soc. Am. A 17, 1192 (2000).

8. S. E. D. Webb, Y. Gu, S. Lévêque-Fort, J. Siegel, M. J. Cole, K. Dowling, R. Jones, P. M. W. French, M. A. A. Neil, R. Jukaitis, L. O. D. Sucharov, T. Wilson, and M. J. Lever, Rev. Sci. Instrum. 73, 1898 (2002). 